\title{
Sprache und Mathematik - theoretische Analysen und empirische Ergebnisse zum Einfluss sprachlicher Fähigkeiten in mathematischen Lern- und Leistungssituationen
}

\author{
Stefan Ufer $\cdot$ Dominik Leiss $\cdot$ Petra Stanat $\cdot$ Hedwig Gasteiger
}

Online publiziert: 9. März 2020

(C) Der/die Autor(en) 2020

Sprache spielt in allen Schulfächern eine zentrale Rolle. Dies gilt auch für das Fach Mathematik (vgl. z. B. Paetsch et al. 2016; Vukovic und Lesaux 2013). Der Zusammenhang zwischen sprachlicher und fachlicher Kompetenz basiert zum einen auf der kommunikativen Funktion von Sprache, die insbesondere im Unterricht zum Tragen kommt. Mathematische Inhalte werden im Unterricht und in Lernsituationen sprachlich vermittelt, etwa durch direkte Instruktion und Erklärungen der Lehrkraft oder im Austausch von Schülerinnen und Schülern untereinander, etwa in Partner- oder Gruppenarbeit. Aber auch in Test- und Prüfungssituationen ist die kommunikative Funktion von Sprache relevant, wenn es zum Beispiel um die Rezeption sprachlich formulierter Aufgabenstellungen oder um die schriftliche Produktion von Lösungen und Lösungswegen geht. Zum anderen hat Sprache in der Mathematik eine kognitivepistemische Funktion, da sie als Werkzeug für das Denken dient. Ohne Sprache ist es kaum möglich, mathematische Konzepte gedanklich zu nutzen und mit ihnen zu operieren, um sie beispielsweise bei der Lösung neuer Probleme anzuwenden (Kempert et al. 2020; Prediger 2017).

\footnotetext{
S. Ufer

Ludwig-Maximilians-Universität München, München, Deutschland

D. Leiss

Leuphana Universität Lüneburg, Lüneburg, Deutschland

P. Stanat

Humboldt-Universität zu Berlin, Berlin, Deutschland

Institut zur Qualitätsentwicklung im Bildungswesen, Berlin, Deutschland

H. Gasteiger $(\bowtie)$

Universität Osnabrück, Osnabrück, Deutschland

E-Mail: hgasteiger@uni-osnabrueck.de
} 
Die Rolle von Sprache für mathematisches Denken und Arbeiten ist bereits seit längerem Gegenstand theoretischer Analysen in der Mathematikdidaktik (z. B. Maier und Schweiger 1999). Trotz der wichtigen Funktionen, die Sprache für das Lehren und Lernen im Fach Mathematik hat, wird sie empirisch jedoch erst seit wenigen Jahren intensiver untersucht. Dabei wird aus unterschiedlichen Perspektiven und mit unterschiedlichen methodischen Ansätzen versucht, die Interdependenzen auszuleuchten, die zwischen Sprache und Mathematik im schulischen Kontext bestehen. In der Mathematikdidaktik verbreitete theoretische Analysen (z.B. Maier und Schweiger 1999; Planas und Schütte 2018), qualitativ-rekonstruktive Herangehensweisen (z. B. Planas 2018) und quantitativ-explorative Studien (z. B. Leiss et al. 2019; Prediger et al. 2015) stehen dabei häufig wenig vernetzt neben quantitativhypothesenprüfenden Quer- und Längsschnittstudien aus der empirischen Bildungsforschung (z. B. Paetsch et al. 2016; Peng und Lin 2019; Powell et al. 2017; Ufer et al. 2013). Noch seltener bearbeitet wird der Bezug zur sprachdidaktischen Forschung (z. B. Becker-Mrotzek et al. 2013; Lucas und Villegas 2011). Ein wesentliches Ziel des Themenhefts bestand deshalb darin, diese verschiedenen Perspektiven sowohl in den Beiträgen als auch im Begutachtungsverfahren konsequent zu berücksichtigen, um den Austausch zwischen den Forschungsansätzen zu intensivieren. Dabei muss jedoch konstatiert werden, dass von den positiv aus dem Begutachtungsprozess hervorgegangenen und nunmehr im Themenheft zusammengeführten Beiträgen die skizzierten Perspektiven nicht in dem Maße ausgewogen vertreten sind, wie es bei der Planung des Heftes vorgesehen war. Eine Ursache hierfür dürfte sicherlich darin zu suchen sein, dass das JMD primär als Publikationsorgan für Kolleginnen und Kollegen der Mathematikdidaktik wahrgenommen wird und beispielsweise Erkenntnisse der empirischen Bildungsforschung, auch wenn sie inhaltlich durchaus eine Passung aufweisen, eher an anderer Stelle zur Publikation eingereicht werden.

Dennoch sind die inhaltlichen und methodischen Zugänge der Studien, über die in den Beiträgen dieses Themenhefts berichtet wird, inhaltlich und methodisch durchaus vielschichtig und betrachten das Thema Sprache im Fachunterricht aus unterschiedlichsten Blickwinkeln. Die Analysen lassen sich unter anderem danach unterscheiden, welche Art von Erkenntnissen bzw. Wissen sie liefern - ob es sich dabei primär um Beschreibungs-, Vorhersage-, Erklärungs- oder Veränderungswissen handelt (vgl. z. B. Bromme et al. 2014). Die Abgrenzung dieser Wissensarten ist zwar nicht immer trennscharf, so dass die meisten Studien Elemente enthalten, die verschiedenen Kategorien zugeordnet werden können. Anhand der zentralen Fragestellungen und der darauf bezogenen Ergebnisse lassen sich die Analysen aber dennoch grob nach dieser Unterscheidung gruppieren.

\section{Beschreibende Analysen}

Zwei der im Themenheft dargestellten Untersuchungen zielen vor allem darauf ab, Beschreibungswissen zu generieren, das sich auf die Rolle von Sprache bei der Bearbeitung spezifischer Lerngegenstände im Fach Mathematik bezieht. Kerstin Tiedemann rekonstruiert in ihrer Studie mit qualitativ-interpretativen Methoden, welche Praktiken des Beschreibens rechenschwache Grundschulkinder bei der Erarbeitung 
des Teilschrittverfahrens im Zahlenraum bis 100 am Rechenrahmen anwenden. Dabei werden drei verschiedene Praktiken des Beschreibens identifiziert, die sich darin unterscheiden wie stark sie charakteristische Einzelelemente des Verfahrens explizit machen. Diese reichen von Beschreibungen, die strukturell mit Materialhandlungen korrespondieren über solche, die zentrale Charakteristika des Verfahrens am Material mit herausheben, bis hin zu Beschreibungen, die diese Charakteristika im Wesentlichen ohne expliziten Bezug zur Materialhandlung enthalten. Die drei identifizierten Praktiken können unterschiedliche epistemische Funktionen im materialbasierten Lernprozess haben. Die Autorin sieht aber auch variierende, mit den Praktiken verbundene sprachliche Anforderungen, die bei der Erarbeitung des mathematischen Inhalts ebenfalls in den Blick genommen werden müssen. Weiterhin verweist sie darauf, dass das Wissen um verschiedene Beschreibungspraktiken für Lehrende hilfreich sein kann, um Beschreibungen gezielt und auch für die Lernenden transparent anzuregen, und dass diese gerade auch in Fördersituationen als Ausgangspunkt für erklärende (statt nur beschreibende) Praktiken genutzt werden sollten.

Auch die qualitative Studie von Susanne Prediger und Dilan Şahin-Gür untersucht die Nutzung von Sprache bei der Bearbeitung mathematischer Aufgabenstellungen. Hierbei geht es um Aufgaben im Bereich der Differenzialrechnung in der Sekundarstufe II und der Fokus liegt auf der syntaktischen Komplexität und ,konzeptuellen Kompaktheit“ der von Schülerinnen und Schülern verwendeten Sprache. In dieser Fallstudie zu Lernprozessen in der Partnerarbeit zeigt sich eine große Varianz in den Verbal- und Nominalphrasen. Dabei scheint eine zunehmende mathematisch-konzeptuelle Präzision entweder mit erhöhter syntaktischer Komplexität oder mit begrifflicher Kondensierung einherzugehen. Dies weist darauf hin, dass zwischen syntaktischer Vereinfachung und semantischer Elaboration ein kompensatorisches Verhältnis in dem Sinne bestehen könnte, dass durch eine begriffliche Kondensierung und die Verwendung entsprechender fachsprachlicher Elemente eine syntaktische Vereinfachung sprachlicher Beschreibungen ermöglicht werden kann. Insgesamt unterstreichen die Ergebnisse der Studie die epistemische Rolle insbesondere konzeptbezogener Fachsprache bei der Arbeit mit mathematischen Konzepten. Die Zusammenschau dieser beiden Beiträge, die primär beschreibend analysieren, wirft insbesondere die Frage auf, inwiefern sich das Spektrum sprachlicher Anforderungen in den Beschreibungspraktiken aus dem Beitrag von Tiedemann anhand der Dimensionen syntaktischer Komplexität und begrifflicher Elaboration beschreiben ließe, die in dem Beitrag von Prediger und Şahin-Gür herausgearbeitet wurden.

\section{Zusammenhangsanalysen}

Bei zwei weiteren Studien steht das Ziel im Vordergrund, Vorhersage- bzw. Erklärungswissen zu ermitteln. Hierzu ist die Analyse von Stefan Ufer und Katrin Bochnik zu zählen, die in einer Längsschnittstudie mit Grundschulkindern der Frage nachgeht, ob fachbezogene sprachliche Kompetenzen zusätzlich zu allgemeinen sprachlichen Kompetenzen zur Vorhersage mathematischer Leistungen beitragen. Die Ergebnisse der Analysen von Daten, die zu zwei Messzeitpunkten im Abstand 
von einem Schuljahr erhoben wurden, bestätigen diese Vermutung, wobei sowohl für den mathematikbezogenen Wortschatz als auch für das mathematikbezogene Textverständnis Effekte identifiziert werden konnten. Die Ergebnisse korrespondieren insofern mit den Hypothesen von Prediger und Şahin-Gür, dass sie Evidenz für den qualitativ herausgearbeiteten Zusammenhang zwischen dem Fachwortschatz, als Mittel zur begrifflichen Kondensierung, und dem Erwerb mathematischer Konzepte an einer größeren Stichprobe und in einem anderen Altersbereich liefern.

Auch die längsschnittliche Studie von Nurit Viesel-Nordmeyer, Ute Ritterfeld und Wilfried Bos beschäftigt sich mit der Frage, inwieweit sprachliche Kompetenzen zur Vorhersage der Entwicklung mathematischer Kompetenz beitragen, wobei Kinder ab dem Vorschulalter über einen Zeitraum von vier Jahren untersucht werden. Die Studie nimmt insofern eine Sonderrolle im Themenheft ein, als sie einerseits nicht explizit fachspezifische Sprachkompetenzen und Sprachhandlungen betrachtet, sondern eher die Rolle von Sprache für das Mathematiklernen im Zusammenhang mit verschiedenen weiteren kognitiven Kompetenzen (zentrale Exekutive, phonologische Schleife, Arbeitsgedächtnis) analysiert. Die Nutzung eines vorhandenen Datensatzes aus dem Nationalen Bildungspanel (NEPS) illustriert einerseits das Potenzial von Reanalysen zur Behandlung von drängenden empirischen Fragen, die sonst aufwändige Primärstudien erfordern würden. Andererseits illustriert der Beitrag aber auch die Herausforderungen bei einem solchen Vorgehen, wenn die theoretische Rahmung nicht direkt das Studiendesign bestimmen konnte. Dabei weisen die Befunde auf ein komplexes Zusammenhangsgefüge zwischen Sprachkompetenzen, kognitiven Kompetenzen und mathematischer Kompetenz hin. Besonders hervorgehoben wird das Grammatikverständnis, das über die gesamte Alterspanne mit der mathematischen Lernentwicklung zusammenzuhängen scheint und dass sich mathematische Kompetenz auch als Prädiktor für spätere kognitive Kompetenzen erweist. Damit werden zumindest erste Hinweise geliefert, wie die drei Kompetenzbereiche (Sprache, Mathematik, kognitive Kompetenzen) zum Beginn des Grundschulalters in ihrer Entwicklung statistisch interagieren.

\section{Analysen zu Wirkmechanismen}

Eine dritte Gruppe von Untersuchungen zielt primär darauf ab, Veränderungswissen zu generieren, also Wirkmechanismen zu untersuchen, mit denen sprachbezogene Prozesse in mathematischen Lern- und Leistungssituationen gefördert bzw. unterstützt werden können. Hierzu gehört die Studie von Anna Noll, Jürgen Roth und Marcus Scholz, die mit einem experimentellen Design überprüft, inwieweit sich zur Reduktion von Lesebarrieren eingesetzte sprachliche Maßnahmen (leichte Sprache) und visuelle Maßnahmen (Piktogramme, Fotos) auf die Lösung von Mathematikaufgaben auswirken. Die Ergebnisse der Studie, die in der Sekundarstufe I durchgeführt wurde, weisen darauf hin, dass zwar nicht leichte Sprache alleine, aber eine Kombination von leichter Sprache und Fotos den Erfolg der Aufgabenbearbeitung erhöhen kann. Eine in diesem Kontext interessante Anschlussfrage wäre, ob eine rein sprachliche Vereinfachung möglich ist, ohne den Text begrifflich stärker zu kondensieren. Wenn dies der Fall wäre, würde dies der von Prediger und Şahin-Gür formulierten 
Hypothese, dass konzeptuelle Kompaktheit und sprachliche Komplexität sich gegenläufig verhalten, teilweise widersprechen. Wenn aber die sprachliche Vereinfachung zu einer Erhöhung der konzeptuellen Komplexität des Textes führt, könnte dies die Notwendigkeit zusätzlicher Informationen - beispielsweise in Form von Fotos erklären. Dass der Einsatz leichter Sprache mit einer Erhöhung der Anzahl der bearbeiteten Aufgaben (unabhängig von der Korrektheit der Bearbeitung) einhergeht, könnte auf affektiv-motivationale Wirkungen solcher Vereinfachungen hinweisen.

In der Studie von Dominik Leiss und Jennifer Plath schließlich geht es um die Frage, inwieweit sich die Unterrichtswahrnehmung und die sprachbasierten Fachleistungen (mathematisches Textaufgabenverständnis und mathematischer Fachwortschatz) bei Schülerinnen und Schülern verändern, deren Lehrkräfte im Rahmen des Programms „Bildung durch Sprache und Schrift“ (BiSS) das Ziel verfolgten, ihren Unterricht in der Sekundarstufe I sprachsensibel zu gestalten. Den Ergebnissen zufolge steigerten sich die untersuchten Fachleistungen im Verlauf des Untersuchungszeitraums von gut einem Schuljahr signifikant, wobei jedoch kein Vergleich mit einer Kontrollgruppe möglich war. Die Einschätzungen der Schülerinnen und Schüler zu sprachbezogenen Merkmalen des Unterrichts haben sich hingegen kaum verändert. Zwar hat sich ihre Haltung gegenüber mathematischen Sprachhandlungen signifikant ungünstig entwickelt, dies jedoch auf einem insgesamt recht hohen Niveau. Auch wenn einer expliziteren Behandlung sprachlicher Praktiken im Unterricht Potenzial für die Unterstützung mathematischer Lernprozesse zugesprochen wird (Götze 2019), deutet sich an, dass dieser Fokus für Lernende (zunächst) eine zusätzliche kognitive Herausforderung darstellen kann. Inwiefern diese Komplexitätssteigerung ,wünschenswert“" in dem Sinne ist, dass sie das Lernen für alle oder bestimmte Lernende unterstützt, muss an dieser Stelle offenbleiben.

\section{Synthese}

Diese zusammenfassende Beschreibung der Studien verdeutlicht, wie vielfältig, komplex und ergiebig das Forschungsfeld Sprache und Mathematik ist. Neben der Unterscheidung nach der Art des Wissens, das sich mit den Studien primär generieren lässt, ließen sich die Analysen anhand einer Vielfalt weiterer Aspekte kategorisieren.

Rolle der Herkunftssprache Interessant ist zunächst, dass in keiner der Untersuchungen zwischen Lernenden mit deutscher und Lernenden mit nichtdeutscher Herkunftssprache unterschieden wird, worauf in der Anfangsphase der Forschung zu sprachlichen Herausforderungen im Fach vielfach ein Hauptaugenmerk lag. Da die Befundlage darauf hinweist, dass sich die fachlichen Schwierigkeiten von Schülerinnen und Schülern mit eingeschränkter Sprachkompetenz nicht in Abhängigkeit davon unterscheiden, ob sie Deutsch als Erst- oder Zweitsprache erworben haben bzw. ob sie ein- oder mehrsprachig aufwachsen (z.B. Heppt 2016; Prediger und Wessel 2018; Ufer et al. 2013), scheint die Bedeutung dieses Forschungsstrangs rückläufig zu sein. Stattdessen liegt der Fokus jetzt stärker auf Mechanismen, die den Einfluss von Sprache vermitteln (Noll, Roth \& Scholz; Prediger \& Şahin-Gür; 
Tiedemann; Ufer \& Bochnik; Viesel-Nordmeyer, Ritterferld \& Bos) und auf den Möglichkeiten, diese Mechanismen gezielt zu beeinflussen, um mathematisches Lernen zu unterstützen (Leiss \& Plath; Noll, Roth \& Scholz).

Funktionen von Sprache Manche Studien nehmen dabei entweder die kommunikative Funktion (z. B. Noll, Roth \& Scholz) oder die kognitive bzw. epistemische Funktion (z.B. Prediger \& Şahin-Gür) überwiegend in den Blick. Bei den meisten anderen Untersuchungen werden diese bei der Beschreibung der Wirkmechanismen weniger klar ausdifferenziert, oder es könnten beide Funktionen eine Rolle spielen. Es stellt sich in diesem Zusammenhang die Frage, ob und wie diese durchaus plausible theoretische Unterscheidung in ihrer Bedeutung für mathematisches Lernen und Leisten konkreter gefasst und einer empirischen Untersuchung zugänglich gemacht werden können. Erst dadurch könnte sich zeigen, inwiefern die Differenzierung zwischen kommunikativer und epistemischer Sprachnutzung bei der Beschreibung und Gestaltung von mathematischen Lern- und Leistungssituationen einen konkreten Mehrwert bietet.

Inhaltsbezogene oder generische Perspektive auf Sprache Einige der Studien nehmen die inhaltsbezogenen Aspekte von Sprachkompetenzen (Ufer \& Bochnik) und Sprachhandlungen (Prediger \& Şahin-Gür; z. T. auch Tiedemann) gezielt in den Blick und untersuchen explizit, welche Rolle fachbezogene Sprache in mathematischen Lernprozessen spielen kann. Bei anderen Beiträgen dürfte diese fachspezifische Perspektive im Rahmen der Studien zwar ebenfalls eine wichtige Rolle gespielt haben, sie war jedoch nicht explizit Teil der Analysen (Leiss \& Plath). Einige Beiträge (Viesel-Nordmeyer, Ritterfeld \& Bos; Noll, Roth \& Scholz) konzeptualisieren die Rolle von Sprache dagegen weitgehend inhaltsunabhängig. Die Befunde zum Spannungsverhältnis begrifflicher Kondensierung und syntaktischer Komplexität von Schüleräußerungen (Prediger \& Şahin-Gür) und zur Bedeutung fachspezifischer Sprachkompetenzen (Ufer \& Bochnik) weisen darauf hin, dass eine inhaltsspezifische Beschreibung sprachbasierter Lernprozesse über eine rein inhaltsübergreifende Perspektive auf Sprache zusätzliche Erklärungskraft haben könnte. Wie sich die Rolle übergreifender sprachlicher Kompetenzen bei der konzeptbezogenen Nutzung von Sprache entfaltet, bleibt jedoch eine offene Frage. Die Antwort hierauf ist insbesondere für die Orchestrierung allgemeiner sprachlicher Unterstützungsmaßnahmen (z. B. durch leichte Sprache: Noll, Roth \& Scholz) und fachbezogener Sprachförderung (z. B. sprachsensibler Fachunterricht: Leiss \& Plath, Arbeit am Fachwortschatz: Schindler et al. 2019) von hoher Relevanz.

Sprachliche Kompetenzen und sprachliche Anforderungen Während sich die meisten Analysen für die Rolle von individuellen Sprachkompetenzen in der Lernentwicklung interessieren, liegt der Fokus einer Studie im Themenheft (Noll, Roth \& Scholz) auf den sprachlichen Anforderungen in Leistungssituationen. Es könnte zielführend sein, beide Forschungsperspektiven in Zukunft noch stärker zu kombinieren. Dies könnte Aufschluss darüber geben, in welchen mathematischen Lern- und Leistungssituationen beispielsweise Lernende mit geringen sprachlichen Kompetenzen besondere Unterstützung benötigen, und inwiefern es punktuell auch notwendig und 
möglich ist, sprachliche Anforderungen zu reduzieren, ohne den mathematischen Gehalt der Situationen zu verändern. Weiterführende Erkenntnisse könnten evtl. gewonnen werden, indem sprachliche und mathematische Anforderungen mathematischer Aufgabenstellungen, sowie sprachliche und mathematische Kompetenzen von Lernenden, etwa in Form von Stufenmodellen, differenzierter beschrieben und aufeinander bezogen werden. Dies könnte den Blick für die Erfordernisse einer kombinierten sprachlichen und fachlichen Förderung im Mathematikunterricht schärfen.

Untersuchte Konstrukte und theoretische Rahmungen Ferner werden in den Studien sowohl auf Seiten der sprachlichen Kompetenz als auch auf Seiten der mathematischen Kompetenz unterschiedliche Aspekte oder Dimensionen untersucht und miteinander in Zusammenhang gebracht. Auch die Annahmen darüber, welche Aspekte und Dimensionen jeweils zusammenhängen, unterscheiden sich, was darauf hindeutet, dass die in diesem Themenheft repräsentierten Forschungsstränge sich überwiegend noch in einer explorativen Phase befinden. Für die weitere Forschung bietet diese Multiperspektivität die Gelegenheit, aber auch die Herausforderung, bedeutsame Konstrukte und Begriffe weiter auszudifferenzieren. Beispiele hierfür sind unterschiedliche Sprachhandlungen (Tiedemann), fachbezogene Sprachkompetenzen (Prediger \& Şahin-Gür; Ufer \& Bochnik) oder das Konzept des sprachsensiblen Fachunterrichts (Leiss \& Plath). Wichtig wäre ferner, Kontrollvariablen zu identifizieren und zu systematisieren, die aus theoretischen Gründen bei der Untersuchung von Sprache im Kontext des Mathematiklernens beachtet werden sollten (z. B. allgemeine kognitive Kompetenzen: Viesel-Nordmeyer, Ritterfeld \& Bos).

\section{Fazit und Ausblick}

Da sich das Forschungsfeld Mathematik und Sprache vor allem in der interdisziplinären Bearbeitung noch in einem recht frühen Stadium befindet, wäre es verfrüht, ein abschließendes Fazit ziehen zu wollen. Hier werden zukünftige Analysen zeigen müssen, welche der Ansätze sich bewähren und zu robusten Ergebnissen führen. Allerdings wäre es wünschenswert, die unterschiedlichen Perspektiven der verschiedenen Autoren, Schulstufen, Unterrichtsfächer und wissenschaftlichen Disziplinen inter- und transdisziplinär zu verbinden, um zu einem zunehmend kohärenten Bild des Phänomens „Sprachnutzung beim Mathematiklernen“ zu kommen. Letztlich ist dabei die Frage zentral, in welcher Weise sprachliche Anforderungen und fachliche Kompetenzen im (Mathematik-)Unterricht zu berücksichtigen sind, damit alle Schülerinnen und Schüler einen möglichst guten Lernerfolg erreichen können. Mit diesem Themenheft sollten einige Bausteine zur Beantwortung dieser Frage gesammelt und Anstöße für die Weiterentwicklung des Forschungsfelds gegeben werden. Wir danken allen Autorinnen und Autoren für ihre Beiträge und freuen uns auf den weiteren Austausch zum Thema Sprache in mathematischen Lern- und Leistungssituationen.

Funding Open Access funding provided by Projekt DEAL.

Open Access Dieser Artikel wird unter der Creative Commons Namensnennung 4.0 International Lizenz veröffentlicht, welche die Nutzung, Vervielfältigung, Bearbeitung, Verbreitung und Wiedergabe in 
jeglichem Medium und Format erlaubt, sofern Sie den/die ursprünglichen Autor(en) und die Quelle ordnungsgemäß nennen, einen Link zur Creative Commons Lizenz beifügen und angeben, ob Änderungen vorgenommen wurden.

Die in diesem Artikel enthaltenen Bilder und sonstiges Drittmaterial unterliegen ebenfalls der genannten Creative Commons Lizenz, sofern sich aus der Abbildungslegende nichts anderes ergibt. Sofern das betreffende Material nicht unter der genannten Creative Commons Lizenz steht und die betreffende Handlung nicht nach gesetzlichen Vorschriften erlaubt ist, ist für die oben aufgeführten Weiterverwendungen des Materials die Einwilligung des jeweiligen Rechteinhabers einzuholen.

Weitere Details zur Lizenz entnehmen Sie bitte der Lizenzinformation auf http://creativecommons.org/ licenses/by/4.0/deed.de.

\section{Literatur}

Becker-Mrotzek, M., Schramm, K., Thürmann, E., \& Vollmer, H. (2013). Sprache im Fach: Sprachlichkeit und fachliches Lernen. Münster: Waxmann.

Bromme, R., Prenzel, M., \& Jäger, M. (2014). Empirische Bildungsforschung und evidenzbasierte Bildungspolitik. Eine Analyse von Anforderungen an die Darstellung, Interpretation und Rezeption empirischer Befunde. Zeitschrift für Erziehungswissenschaft, 17, 3-54.

Götze, D. (2019). Schriftliches Erklären operativer Muster fördern. Journal für Mathematik-Didaktik, $40(1), 95-121$.

Heppt, B. (2016). Verständnis von Bildungssprache bei Kindern mit deutscher und nicht-deutscher Familiensprache. Dissertation an der Humboldt-Universität zu Berlin.

Kempert, S., Schalk, L., \& Saalbach, H. (2019). Sprache als Werkzeug des Lernens: Ein Überblick zu den kommunikativen und kognitiven Funktionen der Sprache und deren Bedeutung für den fachlichen Wissenserwerb. Psychologie in Erziehung und Unterricht, 66, 176-195.

Leiss, D., Plath, J., \& Schwippert, K. (2019). Language and mathematics-key factors influencing the comprehension process in reality-based tasks. Mathematical Thinking and Learning, 21(2), 131-153.

Lucas, T., \& Villegas, A. M. (2011). A framework for preparing linguistically responsive teachers. In T. Lucas (Hrsg.), Teacher preparation for linguistically diverse classrooms (S. 55-72). New York: Routledge.

Maier, H., \& Schweiger, F. (1999). Mathematik und Sprache: Zum Verstehen und Verwenden von Fachsprache im Mathematikunterricht. Wien: oebv + hpt.

Paetsch, J., Radmann, S., Felbrich, A., Lehmann, R., \& Stanat, P. (2016). Sprachkompetenz als Prädiktor mathematischer Kompetenzentwicklung von Kindern deutscher und nicht-deutscher Familiensprache. Zeitschrift für Entwicklungspsychologie und Pädagogische Psychologie, 48, 27-41.

Peng, P., \& Lin, X. (2019). The relation between mathematics vocabulary and mathematics performance among fourth graders. Learning and Individual Differences, 69, 11-21.

Planas, N. (2018). Language as resource: a key notion for understanding the complexity of mathematics learning. Educational Studies in Mathematics, 98(3), 215-229.

Planas, N., \& Schütte, M. (2018). Research frameworks for the study of language in mathematics education. ZDM Mathematics Education, 50(6), 965-974.

Powell, S. R., Driver, M. K., Roberts, G., \& Fall, A. M. (2017). An analysis of the mathematics vocabulary knowledge of third-and fifth-grade students: Connections to general vocabulary and mathematics computation. Learning and Individual Differences, 57, 22-32.

Prediger, S. (2017). Auf sprachliche Heterogenität im Mathematikunterricht vorbereiten - Fokussierte Problemdiagnose und Förderansätze. In J. Leuders, T. Leuders, S. Prediger \& S. Ruwisch (Hrsg.), Mit Heterogenität im Mathematikunterricht umgehen lernen - Konzepte und Perspektiven für eine zentrale Anforderung an die Lehrerbildung (S. 29-40). Wiesbaden: Springer Spektrum.

Prediger, S., \& Wessel, L. (2018). Brauchen mehrsprachige Jugendliche eine andere fach- und sprachintegrierte Förderung als einsprachige? Zeitschrift für Erziehungswissenschaft, 21(2), 361-382.

Prediger, S., Wilhelm, N., Büchter, A., Gürsoy, E., \& Benholz, C. (2015). Sprachkompetenz und Mathematikleistung - Empirische Untersuchung sprachlich bedingter Hürden in den Zentralen Prüfungen 10. Journal für Mathematik-Didaktik, 36(1), 77-104. 
Schindler, V., Moser-Opitz, E., Cadonau-Bieler, M., \& Ritterfeld, U. (2019). Überprüfung und Förderung des mathematischen Fachwortschatzes der Grundschulmathematik - eine empirische Studie. Journal für Mathematik-Didaktik, 40(1), 1-35.

Ufer, S., Reiss, K., \& Mehringer, V. (2013). Sprachstand, soziale Herkunft und Bilingualität: Effekte auf Facetten mathematischer Kompetenz. In M. Becker-Mrotzek, K. Schramm, E. Thürmann \& H. J. Vollmer (Hrsg.), Sprache im Fach (S. 185-202). Münster: Waxmann.

Vukovic, R. K., \& Lesaux, N.K. (2013). The language of mathematics: investigating the ways language counts for children's mathematical development. Journal of Experimental Child Psychology, 115, $227-244$. 